\title{
Anticipation for Efficient Electricity Transmission Network Investments
}

\author{
Vincent Rious, Philippe Dessante, Jean-Michel Glachant
}

\begin{abstract}
This paper proposes a model and preliminary results to evaluate the efficiency of anticipating the connection of power plants with shorter construction duration than the time needed to obtain the right to upgrade the network and finally to do this reinforcement. This evaluation is made in presence of a cost of anticipation related to the study of the project of network investment and to the administrative procedures needed to obtain the building agreement. This model compares a proactive TSO that anticipates the connection of new generators and then the required network reinforcement, with a reactive one that does not make any anticipation but that may then face greater congestion while the network is being reinforced. The efficiency of these behaviors is measured in terms of social cost. We find out that there exists a limit of probability for the connection of generators beyond which a proactive TSO is more efficient than a reactive one. Evaluated on a realistic case of connection, this limit of probability is found quite low, which indicates that the proactive behavior for a TSO shall generally be the optimal one.
\end{abstract}

\section{INTRODUCTION}

$\mathrm{P}_{\mathrm{a}}^{\mathrm{o}}$ OWER generation and transmission are complementary activities that need to be coordinated, to the short-run and to the long term to ensure an optimal use and development of the transmission network.

The coordination between generation and transmission is more difficult in a liberalised power system, not only because these activities are unbundled but also because of the investors' choice for generation technology. The power reform has prompted the generation investors to build mainly power plants that can be built quickly, such as Combined Cycle Gas Turbines or wind farms [1] [2]. At the same time, the right of way of powerlines faces increasing oppositions [3]. These movements increase the time needed to build transmission lines.

These differences in construction duration create uncertainty for the network planning. Indeed, these differences in construction time are all the more detrimental that the generation capacities of these new plants are

Manuscript received April 19, 2008. Vincent Rious gratefully acknowledges the financial and scientific support he received from the French TSO RTE for this work while he was a Ph. D student under the supervision of Prof. Jean-Michel Glachant at both the GRJM Team at the Research Center ADIS - University Paris XI, and at the Energy department at Supélec.

V. Rious and P. Dessante are with Supelec, Gif-sur-Yvette, 91190 France (corresponding author: V. Rious; phone: +33(0)169851 531; fax: +33 (0)169851 539; e-mail: vincent.rious@supelec.fr).

J.-M. Glachant is with University Paris-Sud XI, Fontenay-aux-Roses, 92260 France. significant compared to the transmission lines capacities. The connection of these power plants can thus create congestion.

A solution to this problem could be that the Transmission and System Operator (TSO) anticipates the connection of these new generation plants and the congestions that they may create. By anticipating the connection of generation plants, the TSO can adapt the network planning. To implement this process, the TSO must anticipate the administrative procedures required before the network upgrading. But if the network is not eventually upgraded, this anticipation is costly because of these administrative procedures. The efficiency of anticipating the generation connection and of the required transmission investment thus depends on this cost, on the uncertainty on the effective generation connection and on the required transmission investment.

This paper evaluates the efficiency of the strategy of anticipating the connection of power plants for the TSO in terms of the minimization of the network cost. The question is then to know if it is efficient for such a TSO to plan the development of its network in advance of the request of connection so that there is sufficient planned transmission capacity to accommodate these new generation investments. This problem has been poorly addressed [4].

A model is built in this paper in order to measure the efficiency of anticipating the connection of generation. In this model, the connection of a generator is a probabilistic event and the TSO can choose to anticipate the connections or not, despite this anticipation may be costly if the anticipated event does not eventually occur.

The paper is then organized as follow. In section II, we show that the need to coordinate generation and transmission varies with the considered generation technology. In section III, we present our model to evaluate the efficiency of anticipating the generation connection and the required transmission investment depending on the cost of this anticipation, on the probability of the power plants to connect and on the difference in construction duration between the transmission and generation investments. In section IV, we made a preliminary measure of efficiency of anticipating connection on a realistic example.

\section{A NEED TO COORDINATE TRANSMISSION AND GENERATION THAT VARIES WITH THE GENERATION TECHNOLOGY}

In a liberalized power system where generation and transmission are generally unbundled, the need to coordinate 
these activities varies with the generation technology. Indeed, the time needed to build powerlines can be longer than the time needed to build some generation technologies.

It takes at least five years to build a powerline and on average seven to ten years in Europe [3]. There are two steps to build a powerline. Firstly, the TSO must fulfil the administrative procedures to have the right to build the line. This step to obtain the administrative agreements lasts at least three years. But in practice, it can last five years on average. The second step consists in building the line. This step is quite short, about two years only, and faces few uncertainties. The step to obtain the administrative agreements is then the crucial step for the time between the investment decision and the completion of the project. The uncertainty on building the powerline comes from this period because of the local oppositions to the right of way of the transmission lines, which can result in postponing the line project.

The generation technology induces an important variation of the difference in investment time between transmission and generation. Besides, some generation technologies have an important notional size while they can be quickly built. The connection of the power plants can then create congestion while the TSO has not yet upgraded the network to evacuate this power. This can make the accommodation of these generators more difficult. This difficulty is evaluated in table 1 by the third column that gives the notional size of an installation divided by the time to build it.

TABLE I

TIME NEEDED TO BUILD DIFFERENT GENERATION TECHNOLOGIES [6] [7](RAE2004, DGEMP2003)

\begin{tabular}{|l|c|c|c|}
\hline $\begin{array}{c}\text { Generation } \\
\text { technology }\end{array}$ & $\begin{array}{c}\text { Time } \\
\text { needed to } \\
\text { build (year) }\end{array}$ & $\begin{array}{c}\text { Notional size } \\
\text { (MW) }\end{array}$ & $\begin{array}{c}\text { Size divided by } \\
\text { time to build } \\
\text { (MW/year) }\end{array}$ \\
\hline Combustion turbine & 1 & 40 & 40 \\
\hline Coal & $4-5$ & 150 to $1600^{*}$ & 30 to $400^{*}$ \\
\hline CCG & 2 & 800 & 400 \\
\hline Nuclear & $5-7$ & 1600 & 200 to 300 \\
\hline Hydro & - & - & - \\
\hline Wind onshore & 2 & 25 & 12.5 \\
\hline \multicolumn{1}{|c|}{ offshore } & 2 & 100 & 50 \\
\hline
\end{tabular}

*Depending on technologies

The investment dynamics of the nuclear and coal technologies are similar to the investment dynamics of network investments. The TSO can then deal with their connection when required. To the contrary, the CCGT and the wind farms can be built and connected faster than the network can be modified to accommodate them. Therefore, these new generation units can create important congestion before the TSO can upgrade the network.

This phenomenon becomes very important because these technologies, CCGT and wind farms, are the fastest to be built and are actually the preferred ones in Europe and in the USA (see [1] for CCGT and [2] for wind power).

The time to build CCGT is quite short since it is only about two to three years [5] [6] The notional size of CCGT is 800
MW. It cannot be neglected compared to the transmission capacity of powerlines, between 1000 and $2500 \mathrm{MW}$ for the voltage level where they connect $(400 \mathrm{kV}$ or $225 \mathrm{kV})$.

The massive connection of wind power to the transmission network is also problematic for two reasons. Firstly, compared to the time to upgrade the network, the time to build wind farms is quite short, since it is about two to three years. Secondly, the network must adapt to the massive connection of intermittent power plants. Besides, their massive connection can then require upgrading the transmission network to evacuate the generated power.

Therefore, the problem is the following one: some generation technologies can require important network upgrading whose time to build is quite longer than the time to build these power plants. There may then be congestion between the moment when these generators connect to the network and the moment when the TSO upgrades the facilities.

The TSO can anticipate the connection of these plants and consequently plan the network investment to avoid these congestions. Then it can better deal with the uncertainty coming from the difference in time to build power plants and time to upgrade the network. But this work of anticipation is costly. As a consequence, it is necessary to evaluate the efficiency of anticipating the network investment to accommodate new generators.

\section{A MODEL TO MEASURE THE EFFICIENCY OF ANTICIPATING NETWORK INVESTMENTS}

In order not to limit and even in order to facilitate the development of the types of generators with the shortest time to build, it can be efficient to anticipate their connections. The network can then have sufficient capacity to accommodate them. The TSO can anticipate the connection of these generators and can so study in advance the opportunity of upgrading the network.

Such anticipation does not compel the TSO to invest if it eventually happens unnecessary. Because, once the TSO has obtained the administrative agreements required before building the power line, the TSO can decide to upgrade the network effectively only after the relevant assumptions of the investment project has come true or extremely certain. To the contrary, a TSO whose objective is to maximise the social welfare can decide to cancel a planned investment if the relevant conditions does not eventually happen. The administrative steps needed before the building of the transmission line have then an appreciable option value if the TSO can implement various planning strategies to invest [7].

The TSO can implement two strategies to anticipate transmission reinforcements. $1^{\circ}$ The TSO can be proactive and anticipates the change in the generation mix and location. $2^{\circ}$ The TSO can be reactive and upgrades the network only once he knows where and when the power plants connect. In the reference [4], Sauma and Oren show that, from the point of 
view of the minimization of the expected social cost, the proactive TSO is always more efficient than the reactive one in an uncertain environment. But they implicitly assume that anticipation has no cost while it is costly in reality. And it could be quite expensive [8].

The essential parameters to evaluate the efficiency of the anticipating the generation and transmission investment are then the three following ones: $1^{\circ}$ the cost of anticipating investments, $2^{\circ}$ the difference between the time to build power plants and the time to upgrade the network, and $3^{\circ}$ the probability of connection of generators. Our model will allow measuring the influence of these different elements on the opportunity for the TSO to be proactive. We describe this model below. Next, we will illustrate our results on a representative case of connection.

\section{A. A necessary condition for anticipation to be optimal}

We present here a simplified model where the congestion cost is assumed given and sufficient to require a transmission investment. The problem of calculating the congestion cost is dealt with further detail in section IV of this paper in the realistic case of connection of a power plant. In our modelling, we search for the conditions when it is efficient, from the point of view of the minimization of the expected social cost, to anticipate the connection of power plants whose time to build is shorter that the time to upgrade the network.

We consider the two proactive and reactive behaviors of the TSO that we described just above. To anticipate the connection of new power plants, a proactive TSO does in advance the study of the transmission investment project and the administrative procedures that are required to have the agreements to build the powerline.

A reactive TSO does not study the project nor does he make the administrative procedures in advance. He does these steps only once the generators have effectively asked to connect to the network.

We assume that in a step before the application of our modelling, an expert has highlighted the nodes or areas where generators are more likely to connect and the lines that may experience congestion. It is similar to the approach used in the National Electric Transmission Congestion Study of the U.S. Department of Energy [9]. This phase consists in determining where it will be needed to upgrade the network taking into account the primary energy sources and the areas where the generators will be able to locate. The goal of our modelling is then not to find where to upgrade the network. Our modelling finds the lines whose forecasted constraints are such that it is efficient to anticipate its upgrading and especially to anticipate the long administrative procedures. Anticipating can result in accommodating the considered generation technologies more efficiently and more quickly.

\section{B. Definitions and assumptions}

For each year $y$, we define two types of congestion cost that we note $C U_{y}$ and $C W_{y}$ respectively with and without network reinforcement. Then, whatever the year $y$, the congestion cost without reinforcement is greater than the congestion cost with reinforcement, other saying $C W_{y} \geq C U_{y}$. For a year $y$, the congestion cost $C W_{y}$ or $C U_{y}$ depends only on reinforcing the network, and not on the moment when the network is upgraded. We define two functions of discounted and cumulative congestion cost over several years $d$ with a discount rate $a$. The first function, $C W(d)$ is the total congestion cost discounted during $d$ years before upgrading the network. The second function, $C U(d)$ is the total congestion cost discounted during ten years after the network being upgraded the year $d$. The avoided costs thanks to the reinforcement of the network are evaluated only over ten years for two reasons. Firstly it is difficult to know accurately the state of a power system beyond ten years. Secondly discounting considerably decreases the costs beyond this temporal horizon. $C W(d)$ and $C U(d)$ can be expressed as functions of $C W_{y}$ and $C U_{y}$ as follow:

$$
C W(d)=\sum_{y=1}^{d} \frac{C W_{y}}{(1+a)^{y}} \text { and } C U(d)=\sum_{y=d+1}^{d+10} \frac{C U_{y}}{(1+a)^{y}}
$$

Fig. 1 exemplifies the two sums $C W(d)$ and $C U(d)$. For illustrative reasons, we assume that the terms $C W_{y} /(1+a)^{y}$ and $C U_{y} /(1+a)^{y}$ increase linearly with time. $C W(d)$ corresponds to the grey trapezoid and $C U(d)$ corresponds to the black trapezoid.

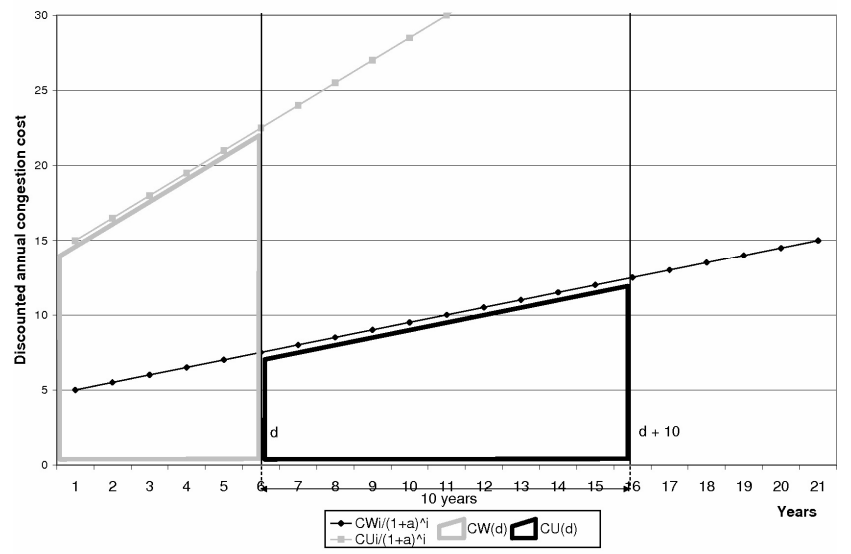

Fig. 1. Definition of $\mathrm{CW}(\mathrm{d})$ and $\mathrm{CU}(\mathrm{d})$.

We assume that the network must be reinforced as soon as a power plant connects. That is to say that the cost saved by the network upgrading as soon as the generator connects is greater than the related transmission investment cost. Fig. 2 illustrates the cost saved by upgrading the network as a function of $C W(10)$ and $C U(0)$.

The equation (2) links $C W(10), C U(0)$ and the investment 
$\operatorname{cost} I$.

$$
C W(10)-C U(0) \geq I
$$

With $C W(10)-C U(0)=\sum_{i=1}^{10} \frac{C W_{i}-C U_{i}}{(1+a)^{i}}$

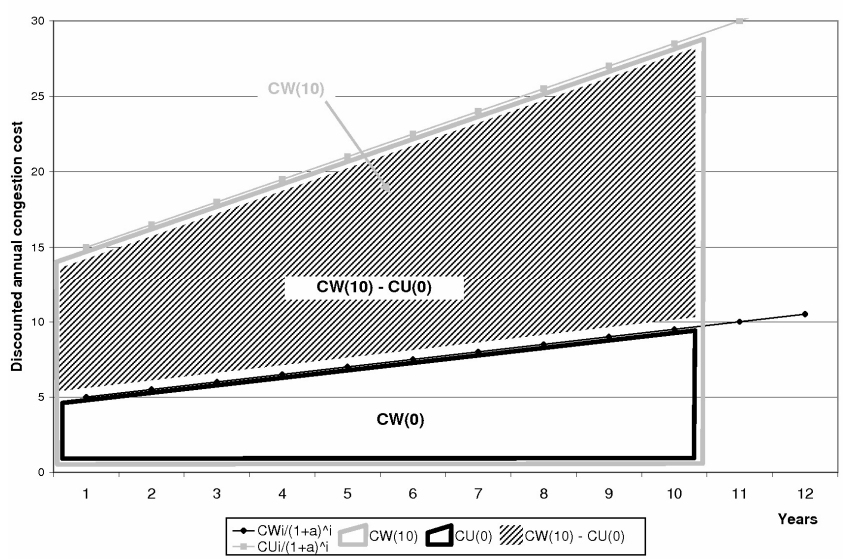

Fig. 2. Cost saved by upgrading the network as a function of $C W(10)$ and $C U(0)$. The greyed area stands for this saved cost.

The instant of reference for discounting the cost over time is chosen such that the most conservative condition about the efficiency of a proactive TSO is obtained. This instant is then the moment when the generation investment begins to produce power. This convention degrades the advantage of discounting for the strategy of anticipating compared to the strategy of not anticipating. Indeed, if the beginning of the administrative procedures has been chosen as the instant of reference for discounting, the discounting of cost would have mechanically decreased the cost of network investment. By taking the beginning of production of the power plant as a time reference for discounting, this effect is avoided.

\section{Expected cost of the network for a reactive TSO}

A reactive TSO does not anticipate the connection of generators. It studies the network upgrading only once the generator has invested. The network investment to evacuate this power is ready to serve only $d$ years after the connection of the generation unit, where $d$ is the difference between the time to build a power plant and the time to upgrade the network. Fig. 3 exemplifies this sequence of the generation and transmission investments.

The generator can connect to the network with a probability $p$ (and so does not connect with a probability of 1-p). This uncertainty is not intrinsically quantifiable. However it is possible to attribute it a subjective value to evaluate the robustness of the study framework. This approach stimulates a dialog with the other stakeholders of the power system and creates a shared anticipation of the evolution of the system [10]. Besides, the sooner the residents are involved in the transmission investment process, the easier the powerline would be built [11] [12].

If the generator connects, the system must successively support:

- $\quad C W(d)$, the total congestion cost discounted for $d$ years, while the TSO is upgrading the network

- $\quad C U(d)$, the residual total congestion cost discounted for ten years after upgrading the network, that is to say $d$ years after the connection of the generator,

- $\quad I /(1+a)^{d}$, the discounted cost of this upgrading $d$ years after the beginning of our study (corresponding to the moment when the generator is ready to generate power)

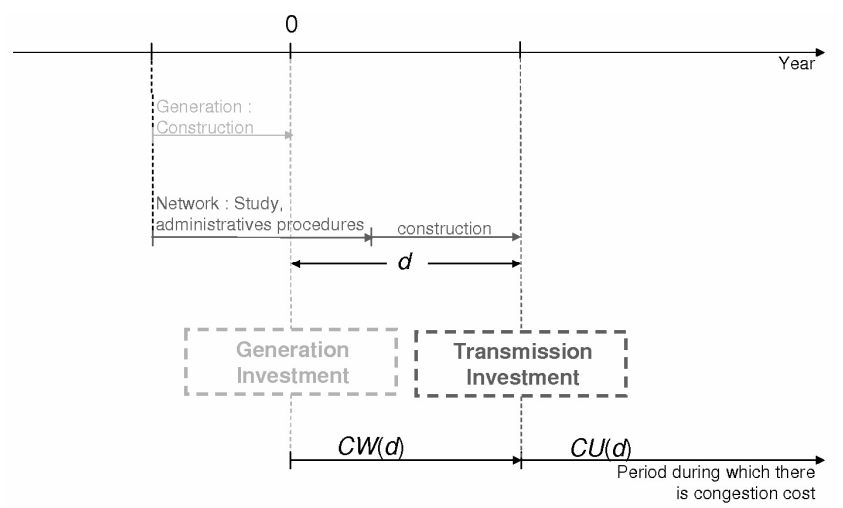

Fig. 3. Sequence of the generation and transmission investment with a reactive TSO. The timeframe of generation is in grey while the timeframe of transmission is in black. While the power plant has already connected and the network has no yet been upgraded, there is congestion for $d$ years.

If the generator does not connect, the network investment cost and the congestion cost to the TSO is null. Table 2 summarises these two cases.

TABLE II

THE COSTS FACED BY A REACTIVE TSO

\begin{tabular}{|l|c|c|c|}
\hline TSO & $\begin{array}{c}\text { invests } \\
\text { Probability } \boldsymbol{p}\end{array}$ & $\begin{array}{c}\text { does not invest } \\
\text { Probability } \mathbf{1 - p}\end{array}$ & $\begin{array}{c}\text { Expected social } \\
\text { cost }\end{array}$ \\
\hline $\begin{array}{l}\text { waits for the } \\
\text { connection of } \\
\text { the power } \\
\text { plant before } \\
\text { studying and } \\
\text { upgrading the } \\
\text { network }\end{array}$ & $\begin{array}{c}C S(d)+C R(d) \\
+I /(1+a)^{d}\end{array}$ & $0+0$ & $E\left[C_{\text {reactiverso }}(p)\right]$ \\
\hline
\end{tabular}

\section{Expected cost of the network for a proactive TSO}

A proactive TSO anticipates the connection of the generator. He studies the network upgrading and asks for the administrative agreements to build the powerline (without building it) before the connection of the power station. The network is upgraded only once the power plant is being effectively connected. Fig. 4 illustrates the sequence of the generation and transmission investments.

The generator can connect with a probability $p$ (and so does 
not connect with a probability 1-p). In case the generator connects to the network, the system must successively support:

- $\quad C U(0)$, the residual total congestion cost discounted for ten years after the power plant connecting and the network upgrading

- $\quad$ and $I$, the upgrading cost.

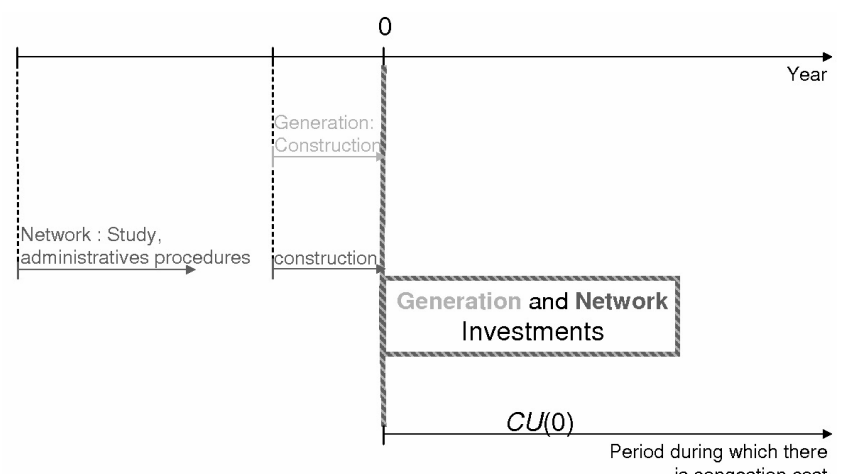
is congestion cost

Fig. 4 Sequence of the generation and transmission investment with a proactive TSO. The use of the new transmission line and of the new power plant begins at the same time because the TSO has anticipated the network upgrading.

In case of the generator not connecting, the congestion cost is null and the cost to anticipate linked to the non realization of the anticipated event is a share $\alpha$ of the total investment transmission cost. Indeed, the transmission investment is not done but the preliminary steps are however realized. The cost $\alpha . I$ includes not only the cost to anticipate to the TSO but also the cost born by the local authorities involved in the process of administrative agreements. Moreover, the cost $\alpha . I$ takes into account the a posteriori discounting of the cost to anticipate. We assume the cost to anticipate $\alpha . I$ is proportional to the investment cost because a power line faces all the more oppositions that it is longer and goes through a wider area.

Table 3 summarises these cost.

TABLE III

THE COSTS FACED BY A PROACTIVE TSO

\begin{tabular}{|c|c|c|c|}
\hline TSO Generator & $\begin{array}{c}\text { Invests } \\
\text { Probability } p\end{array}$ & $\begin{array}{l}\text { Does not invest } \\
\text { Probability 1-p } \\
\end{array}$ & Expected social cost \\
\hline $\begin{array}{l}\text { Studies beforehand } \\
\text { the installation of } \\
\text { the power plant } \\
\text { and invests at the } \\
\text { same time as the } \\
\text { generator }\end{array}$ & $I+C R(0)$ & $\alpha . I+0$ & $\begin{array}{l}E\left[C_{\text {proactive ero }}(p)\right] \\
=p[I+C R(0)]+(1-p) \alpha\end{array}$ \\
\hline
\end{tabular}

\section{E. Condition for a proactive TSO to be efficient}

We are searching for the necessary and sufficient condition for the proactive TSO to be more efficient than the reactive one from the point of view of the minimization of the expected social cost.

This condition links the cost $\alpha$ to anticipate, the probability $p$ to connect a power plant and the difference $d$ in time to build a power plant and a power line such that the equation 3 is respected.

$$
E\left[C_{\text {proactiveTSO }}(p)\right] \leq E\left[C_{\text {reactiveTSO }}(p)\right]
$$

The equation 4 equivalently expresses this relation.

$$
\left.\alpha \leq p \mid(1+a)^{-d}+\alpha-1+(C W(d)+C U(d)-C W(0)) / I\right]
$$

To interpret this formula, we consider the case of equality of the equation 4 and then define the limit of probability or "probability limit" $p_{\text {lim }}$ to connect a power plant.

$$
p_{\lim }=\frac{\alpha}{\left[(1+a)^{-d}+\alpha-1+[C W(d)+C U(d)-C W(0)] / I\right]}
$$

If the probability to connect a power plant is greater than $p_{\text {lim }}$, then the proactive TSO is more efficient than the reactive one. To the contrary, if the probability of connection $p$ is less than $p_{\text {lim }}$, then the reactive TSO is more efficient than the proactive TSO. The equivalence between the equations 3 and 4 then shows that the strategy of anticipation is all the more efficient that the probability $p_{\lim }$ is small. The interpretation of the equation 4 also consists in evaluating how the probability limit $p_{\text {lim }}$ varies with the cost $\alpha$ for anticipating and the difference $d$ in time to build a generation investment and a transmission one. For a given cost $\alpha$ for anticipating, $p_{\text {lim }}$ decreases when the difference $d$ between the time to build a power plant and a power line increases. This is because the congestion cost generally increases more quickly than the gain from postponing the network investment and its discounting. For a given difference $d, p_{\text {lim }}$ increases when the cost $\alpha$ to anticipate increases.

\section{ILLUSTRATION OF PROACTIVE AND REACTIVE TSOS}

Now we illustrate the equation 4 on the concrete case of connection of a power plant. We can determine the combinations of parameters where the proactive TSO is more efficient than the reactive one in this case.

We apply the criterion of the equation 4 on the simple case of the connection of a power plant on a two node network. The figure 5 is realistic because the connection of a power plant (CCGT or windfarm) raises problems mainly because these power plants are far from the load centres.

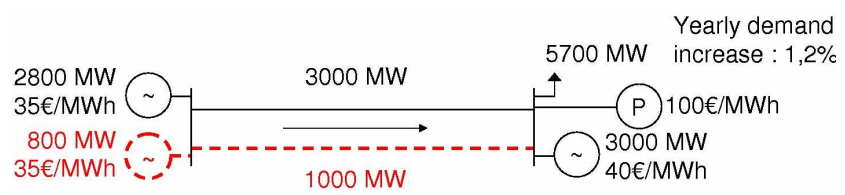

Fig. 5 System to test the strategy to anticipate the connection of a power plant. The load and most of generation are to the east. These power stations to the east are quite expensive, $40 € / \mathrm{MWh}$ for the first $3000 \mathrm{MW}$ and 
$100 € / \mathrm{MWh}$ after. The power plants to the west are less expensive, only $35 € / \mathrm{MWh}$.

A new generator wish to connect a $800 \mathrm{MW}$ power plant to the west. To evacuate the power of this new power plant, it is necessary to add a $1000 \mathrm{MW}$ network upgrading that costs 100 million euros. We assume that the situation of Fig. 5 is representative of the 8760 hours of the year. The investments are dashed on this figure.

First of all, we draw $p_{\text {lim }}$ the "probability limit" to connect a power plant. When the effective probability is above the probability, the proactive TSO is more efficient for a given cost $\alpha$ to anticipate (equal to $10 \%$ of the considered network investment). The "probability limit" can be defined as a function of the difference $d$ between the time of building a power plant and the time of building a powerline. Fig. 6 illustrates this function.

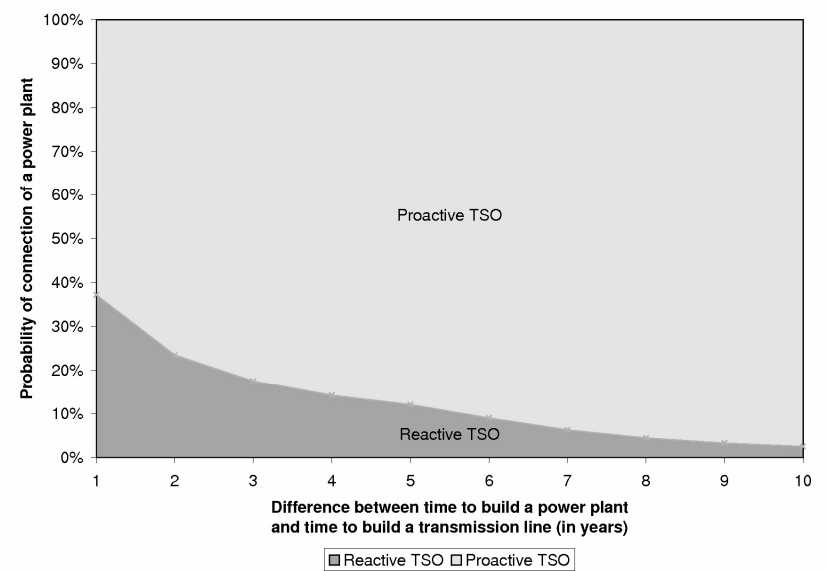

Fig. 6. "Probability limit" of connection beyond which the TSO must be proactive depending on the difference of temporal dynamics $d$ between generation and transmission investment

Above the curve associating the probability limit with the difference in dynamics of investments, the TSO being proactive is the optimal behavior. Under this curve, the TSO being reactive is the optimal behavior. On Fig. 6, we notice that for the considered case, the probability limit decreases as the difference in dynamics increases. We find again that the TSO has little interest in anticipating the connection if the temporal rhythms of these two complementary investments are near each other. That is to say that the time of building a network investment is short or if the time of building a generation investment is long. For the connection of a CCGT or a windfarm, this difference in time is at best of three to four years (see section II). For such a difference, the probability limit of connection is around 15 to $20 \%$. This weak value is already significant to justify the anticipation of the network reinforcement.

\section{CONCLUSION}

This paper presents a model to evaluate the efficiency of the strategy of anticipating the connection of power plants to the network for the TSO in terms of the minimization of the network cost. This model has permitted to see on a simple but realistic example that it can be quite efficient for a TSO to anticipate the connection of power plants. He can then plan the reinforcement of the network in advance and reduce congestion on the network. Taking into account the interest of investors for generation technology with short time to build, the proactive behavior of the TSO can facilitate the connection of these types of power plant and increase the market entries.

However, the study proposed in here is only a preliminary one. We assume that the cost of anticipating is know whereas the planner does not know in advance the cost of anticipating the reinforcement, that is to say the cost of the administrative agreements needed to build the reinforcement. It is indeed difficult to rely on historical data for the cost of anticipation, because the powerlines face more and local oppositions. A sensitivity analysis of the probability limit to the cost of anticipating of the investment should then be one of the future researches of this work. Besides, we could duplicate this study for a windfarm with a given generation duration curve. Eventually, regulatory actions should be studied to incentivise the TSO to anticipate investment if it is efficient. This is still an opened question that is still to be answered.

\section{REFERENCES}

[1] JM. Glachant, "Generation technology mix in competitive electricity markets" in Investments frame and incentives in the electrical industry, Lévêque F. (ed), 2006. Kluwer

[2] ETSO, "European Wind Integration Study (EWIS). Towards a Successful Integration of Wind Power into European Electricity Grids". Final Reports, $15^{\text {th }}$ January 2007. www.etso-net.org

[3] ETSO, "An Overview of Current Cross-border Congestion Management Methods in Europe". May, 2006. www.etso-net.org

[4] E. Sauma and S. Oren, "Proactive planning and valuation of transmission investments in restructured electricity markets", Journal of Regulatory Economics, 30 (3), 2007, 261-290

[5] Royal Academy of Engineering (RAE), The cost of generating electricity, London, March 2004

[6] DGEMP, Coûts de référence de la production électrique, Ministère de l'Économie, des Finances et de l'Industrie, Paris, December 2003

[7] G. Boyle, G. Guthrie and R. Meade, "Real options and transmission investment: the New Zealand Grid Investment Test", ISCR working paper, 2006, www.iscr.org.nz

[8] G. Christiner, "VERBUND-Austrian Power Grid. Austria Completing the $380 \mathrm{kV}$-Ring. Authorization Procedures and Major Problems", CEER Electricity Infrastructure Workshop, 13 February 2007, Bruxelles

[9] U.S. Department of Energy (USDoE), National Electric Transmission Congestion Study, August 2006, www.nietc.anl.gov/documents/docs/Congestion_Study_2006-9MB.pd $\underline{\mathrm{f}}$

[10] J. Bråten, "Estimating benefits. Uncertainty, scenario design and the analysis of benefits", European Transmission Investment Conference, $18-19^{\text {th }}$ October 2004, Copenhague, Danemark

[11] R. Hughes, "Environmental Impact Assessment and Stakeholder Involvement", International Institute for Environment and Development, 2000, www.iied.org/pubs/pdf/full/7789IIED.pdf

[12] J. MacLaren Loring, "Wind energy planning in England, Wales and Denmark: factors influencing project success", Energy Policy, 35(4), 2007, 2648-2660 